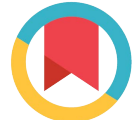

Check for updates

DOI: $10.21859 / \mathrm{ijcp}-03032$

\title{
Advanced Technologies in the Quantification of Mitral Valve
}

\author{
Mersedeh Karvandi ${ }^{1,}$ \\ ${ }^{1}$ Echo Lab, Taleghani Hospital, Shahid Beheshti University of Medical \\ Sciences, Tehran, Iran \\ ${ }^{*}$ Corresponding author: Mersedeh Karvandi, Echo Lab, Taleghani Hospital, \\ Shahid Beheshti University of Medical Sciences, Tehran, Iran. E-mail: \\ mr.karvandi@sbmu.ac.ir
}

\begin{tabular}{|c|c|}
\hline $\begin{array}{l}\text { Submitted: } 10-08-2018 \\
\text { Accented: } 31-08-2018\end{array}$ & \multirow{8}{*}{$\begin{array}{l}\text { Abstract } \\
\text { The current study aimed at conducting an educational survey on the mitral valve using } \\
\text { advanced technologies eSie Valve }{ }^{\circledast} \text { and Q-Lab in echocardiography machines and also } \\
\text { those mitral valve geometrical parameter measurements that can be easily calculated } \\
\text { with MATLAB software offline in a personal computer as an echocardiographic desk } \\
\text { for further information and recommendation before mitral valve repairs or other } \\
\text { treatments. }\end{array}$} \\
\hline Keywords: & \\
\hline Mitral Valve Indices & \\
\hline Echocardiography Imaging & \\
\hline eSie Valve & \\
\hline Q-Lab & \\
\hline Echocardiographic Desk & \\
\hline $\begin{array}{l}\text { (C) 2018. International Journal } \\
\text { of Cardiovascular Practice. }\end{array}$ & \\
\hline
\end{tabular}

\section{INTRODUCTION}

The mechanics of the mitral valve leaflet as nonlinear, inflexible, and anisotropic soft tissue consequence from a combined reaction of many mathematical/physical indices. In the past decade, partial differential numerical modeling of complete heart valves made a good understanding in heart valve surgery, a design of prosthetic valve replacements, and also a general consideration of healthy and abnormal cardiac function. Such a model should be based on a precise description of the automated assessable behavior of the valve. Echocardiography is the best method for the morphology of the heart valves. The practical use of ultrasound techniques is straightforward and its quantitation can be simplified. Echocardiography suggests not only live images of the beating heart for inspection, but also the ability to perform measurable analysis on fixed frames per a cardiac cycle and, more recently, dynamic quantification from moving images. Geometrical measurement analyses play the main roles to achieve the mitral valve ailments. They are used to make the best understanding of valve anatomy, pathology, function, and the structure of the mitral valve diseases. Echocardiographic techniques are the best methods to calculate the quantitative data that are comparable to straight anatomic measurements and surgical tasks.

\section{Methods to Quantify Mitral Valve}

The current study was divided into three main phases in order to quantify mitral valve form, structure, and function as follows:

1- Geometrical mitral valve parameters

2- eSie Valve and Q-Lab package (applied to mitral valve parameters measurements)

3- Utilizing mathematical equation and MATLAB software (a simple echocardiographic desk) (to measure the geometrical mitral valve variables offline based on $2 \mathrm{D}$ or $3 \mathrm{D}$ conventional echocardiographic images)

\section{Geometrical Mitral Valve Parameters}

The modeling techniques proposed in the current study were intended to show the role of geometrical parameters (Figs 1 and 2) of the mitral valve to gain a better understanding of the MV and assist them in obtaining a reliable orientation in order to choose treatment strategies and plan surgical interventions. 


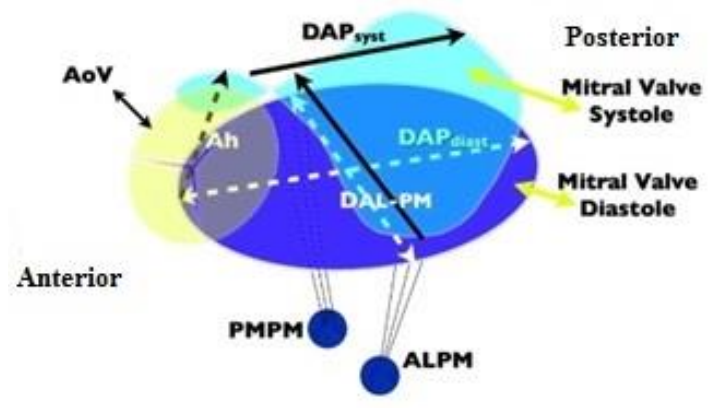

Figure 1: A Schematic Shape of Mitral Valve Components; the Blue Region is in the Mitral Valve Diastole. The Green Region shows the Mitral Valve in Systolic Phase. The Intercommissural Diameter (DAL-PM) (Normal Range 18.7 mm (16.5-19.7); AH, Annular Height (Normal Range $4.4 \mathrm{~mm}$ (3.6-5.4); DAP, Anteroposterior Diameter (Normal Range $16.4 \mathrm{~mm}$ (15.1-17.8))

To study the bending behavior of the mitral valve leaflets, there are several angel dependent variables such as the anteroposterior at the coaptation level in systole (normal range 140 (127-149), the anteroposterior at the commissural level in systole (normal range 126 (113$137)$, and the anteroposterior at the commissural level in diastole (normal range 115 (104-129); however, the anterior leaflet bending angle (sometimes called anterolateral angle) in systole (normal range 119 (106130 ) and diastole (normal range 105 (97-113) should also be considered [1]. The other common quantitative mitral valve parameters are annular height (normal range $4.4 \mathrm{~mm}$ (3.6-5.4), coaptation length (normal range $29.9 \pm 6.6 \mathrm{~mm}$ ), coaptation depth, tending height (normal range $1.59 \mathrm{~mm}$ (1.1-2.24), tending volume (normal range $0.29 \mathrm{~mL}(0.12-0.41)$, posterior prolapse height (normal range $0.7 \mathrm{~mm}$ (039-1.14), and posterior prolapse volume (normal range $0.05 \mathrm{~mL}(0-0.21)[2,3]$.

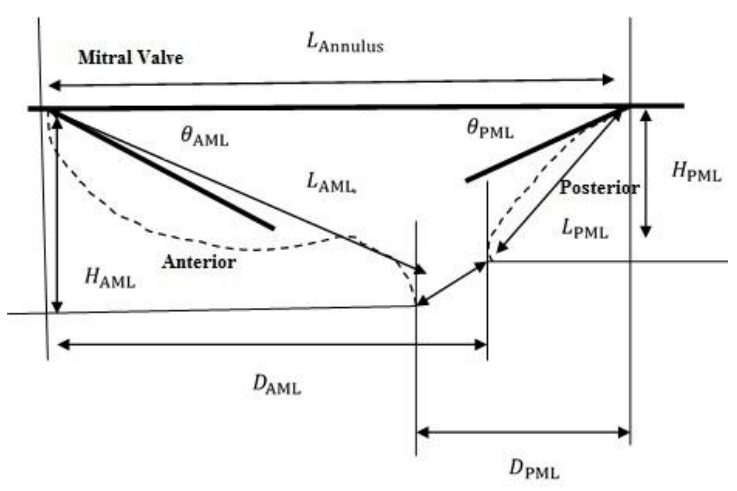

Figure 2: A Schematic Design of the Geometrical Parameters of the Mitral Valve

\section{The Mitral-aortic Angles}

The angle between the mitral and aortic annuli (normal range $\left.118^{\circ} \pm 8^{\circ}\right)($ Fig 3 ) and the angle between the intersection of lines passing through the midpoints of the mitral and aortic valves (normal range $60^{\circ} \pm 6^{\circ}$ ) (Fig 3) are the two very important angles to help predicting the risk of systolic anterior motion of the mitral leaflets [4].

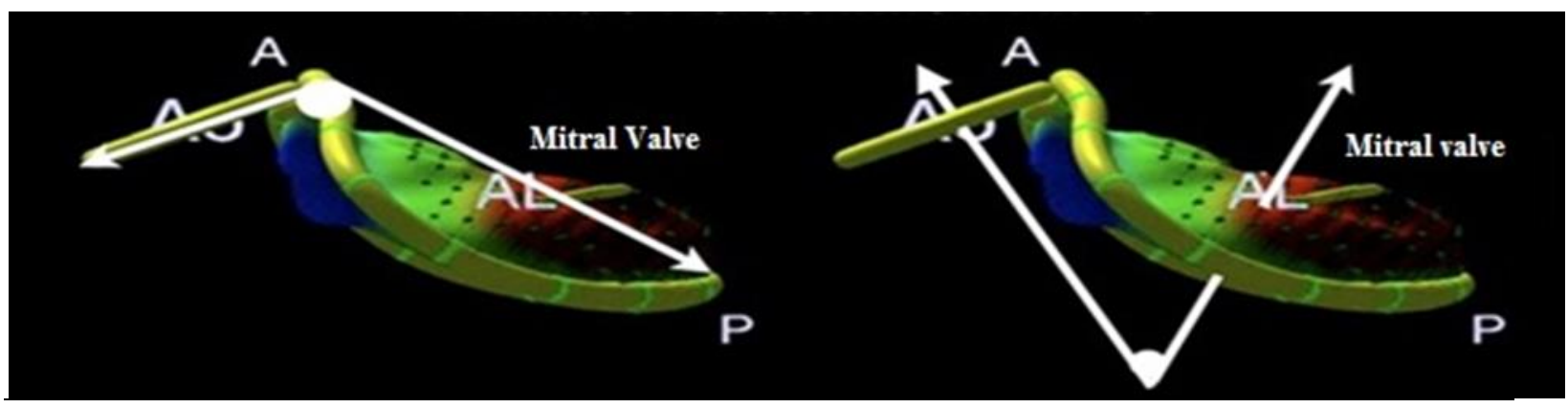

Figure 3: Mitral-Aortic Angles

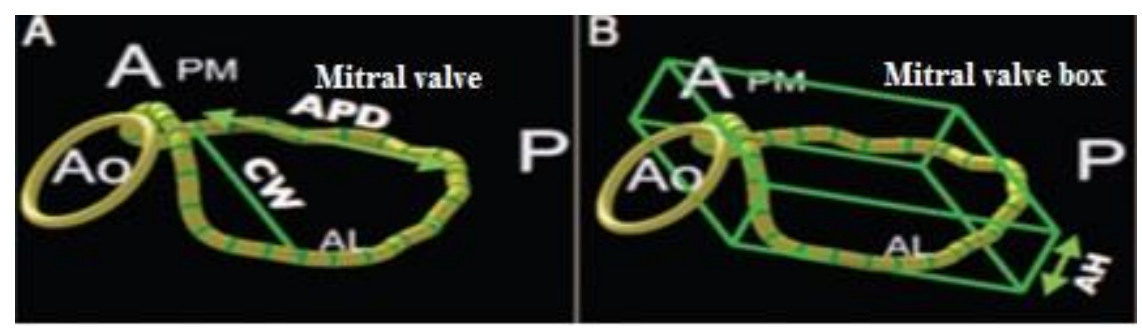

Figure 4: Annular Height and Commissural Width to Provide AHCWR Index

\section{Annular Height to Commissural Width Ratio (AHCWR)}

The saddle shape of the mitral valve annulus can be evaluated/ interpreted by the annular height $(\mathrm{AH})$ (7.9 $\pm 1.9 \mathrm{~mm}$ ) to commissural width ( $\mathrm{CW}$ ) (normal range $33 \pm 3.7 \mathrm{~mm}$ ) ratio of the mitral valve named AHCWR (normal range $23.7 \% \pm 5.4 \%$ ) (Fig 4) [4]. 


\section{Circularity Index}

It is a ratio derived from AP diameter (the distance between the anterior and posterior landmarks) and ALPM diameter (distance between anterolateral and posteromedial landmarks) and is automatically generated for normal range $(0.90 \% \pm 0.1 \%)$ (Fig 5) [5].

\section{The eSie Valve and Q-Lab Package}

The eSie Valve and Q-Lab package rely on an advanced machine learning technology to professionally assess a complete model of the mitral and aortic valves using $2 \mathrm{D}$ or 3D transesophageal echocardiographic (TEE) images. Naturally, a surface model of the valves is fixed to a TEE image using the knowledge learned from a large database of annotated images. For dynamic analysis, a tracking algorithm is employed to estimate a moving valve model throughout the cardiac cycle for quantitative valve analyses [6].

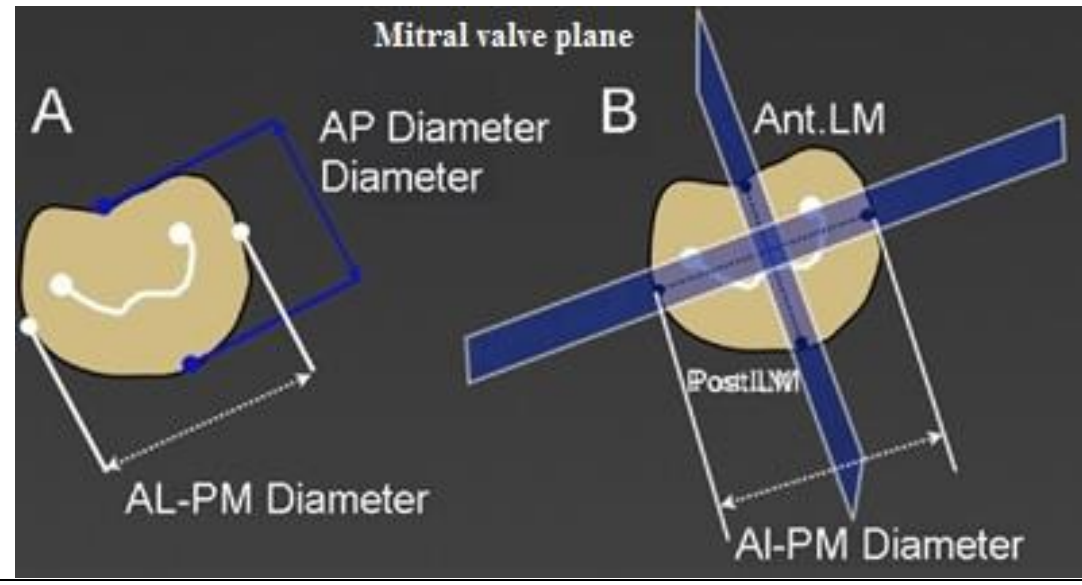

Figure 5: Parameters Measured for the Circulatory Index

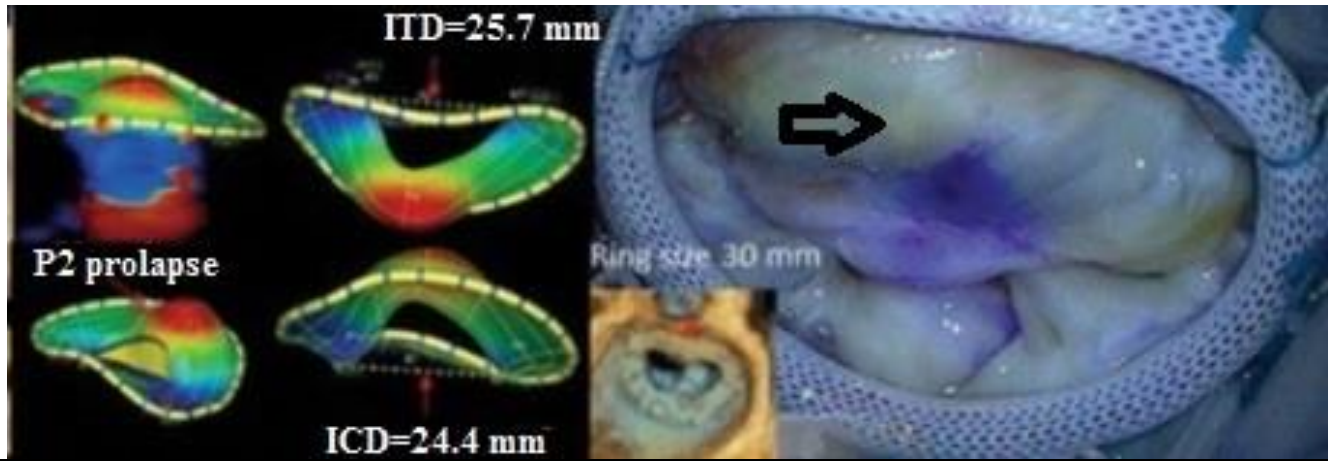

Figure 6: The eSie Valves Package Models a Valve; a Mitral Valve with Severe Posterior Prolapse with a clearly identified P2 Prolapse.

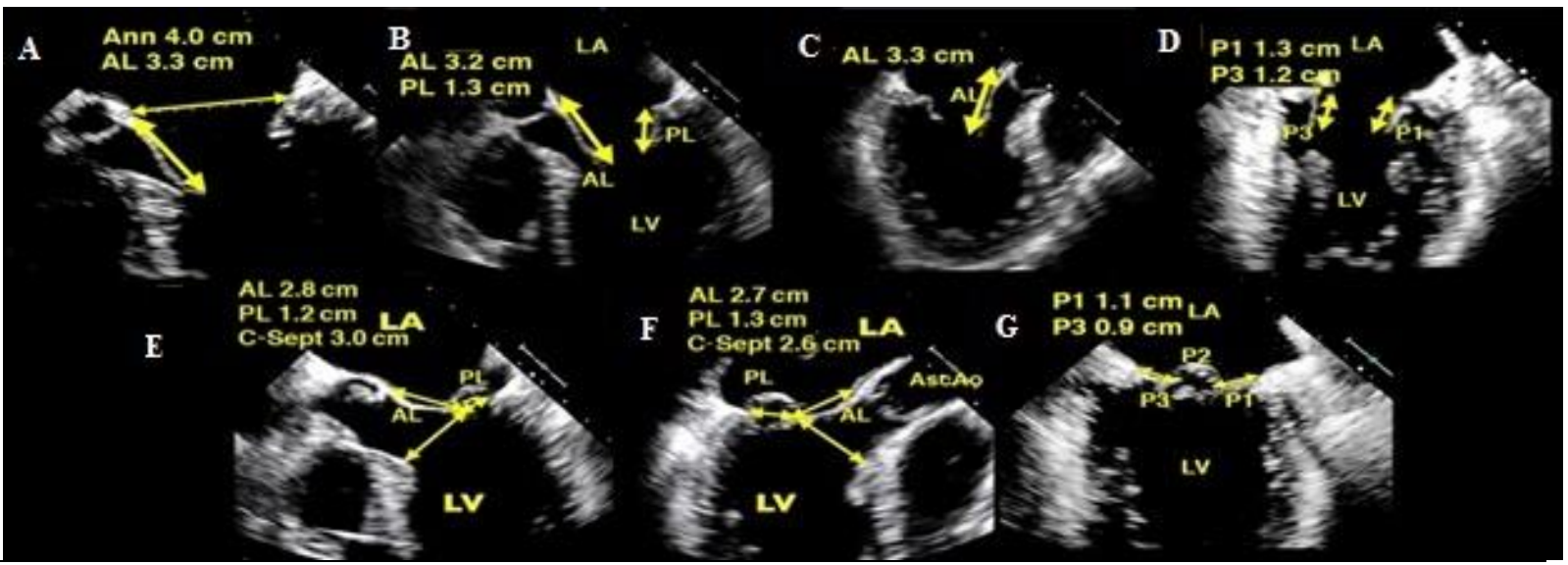

Figure 7: Measurements in Q-Lab: Figures A, B, C, and D are acquired from the Midesophageal 4-/5-Chamber, the LAX, and the Two-chamber Views throughout Ventricular Diastole. Figures E, F, and G are the Consistent Figures Achieved during Ventricular Systole. For this Patient, the Risk of SAM is considered low based on a C-Sept distance $>2.5 \mathrm{~cm}$ and an AL/PL ratio $>1.0$ with Relatively Small P1 and P3 $(<1.4 \mathrm{~cm})$. Ann, Annulus; AL, Anterior Leaflet; PL, Posterior leaflet; LA, Left Atrium; LV, Left Ventricle; C-Sept, the Distance between the Coaptation Point and Ventricular Septum; P1, P2, P3, 3 Scallops of the Posterior Leaflet 


\section{Each Valve is Parameterized by Three Levels of Details}

The first one expresses the region of interest (ROI), where the valve is situated within the image, specifically valve position (center of the ROI), scale (size of the ROI), and orientation. The second, key anatomical points are then defined within the ROI (second level of details). These points are anatomically unique and can be recognized in the images. In the third level of details, symbolized surface models of the valve (mitral leaflets) pass through the points that all mentioned measurements can be calculated automatically from the valve model (e g, A1 area: $159.8 \mathrm{~mm}^{2}$, A2 area: 264.8 $\mathrm{mm}^{2}$, and $\mathrm{A} 3$ area: $136.5 \mathrm{~mm}^{2}$ ). With the eSie Valve and Q-Lab package, the mitral annulus circumference is also a good predictor for ring size, along with intercommissural distance $(\mathrm{r}=0.83$ and 0.80 respectively, all measurements were indexed by body surface area) (Fig 6 and 7) [7-14].

3- Utilizing mathematical equation and MATLAB software (a simple echocardiographic desk)
By utilizing a mathematical equation and MATLAB software, the geometrical mitral valve variables can be measured based on $2 \mathrm{D}$ conventional echocardiographic images.

Tip and tricks to present an echocardiographic image inside the MATLAB software:

First the MATLAB software should be opened; then the command pic = imread (") written down in the command window. This command can be used to read a photo from the computer. The image address, image name, and photo extensions should be written in parentheses. For example, if the echocardiographic image is in the following address: $\mathrm{C}: \backslash$ Users $\backslash$ USER $\backslash$ Desktop $\backslash$ echo image 1.jpg, this command should be written as: pic = imread ('C: \Users \USER $\backslash$ Desktop $\backslash$ echo image 1.jpg'); finally, the image should be called by imshow (pic) command, and then saved by the program and clicking the RUN button. A new window is opened and the searched image is displayed. The distances of the image can be edited or measured by the menus above this observed image (Fig 8).
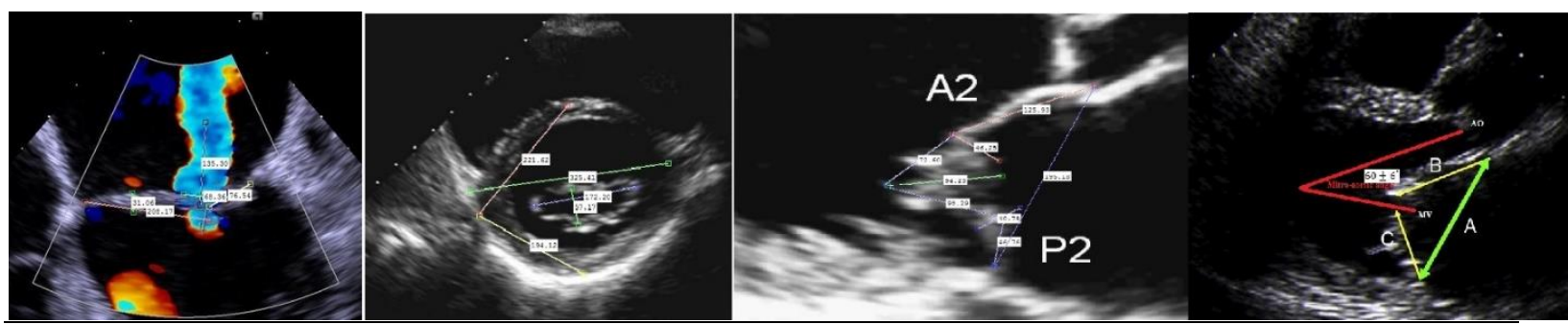

Figure 8: Mitral Valve Measurements Based on Echocardiographic Images as the Inputs in MATLAB Software
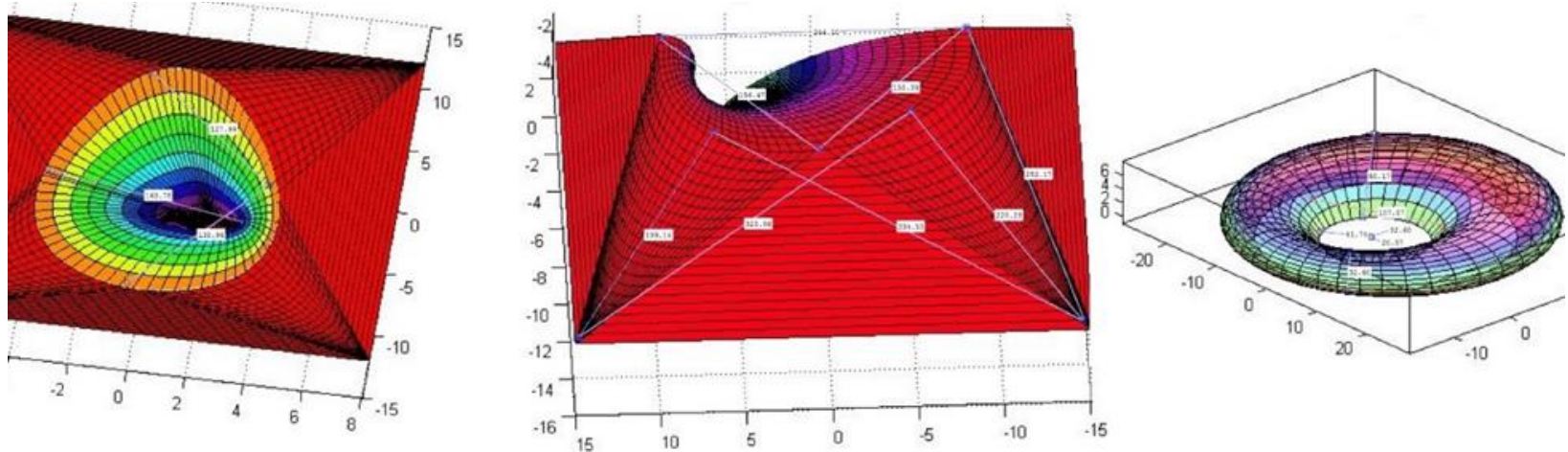

Figure 9: 3D Reconstructions/Measurements of a Mitral Valve Based on Mathematical Equations and the Geometrical Mitral Valve Parameter Measurements in MATLAB Software

Afterward, when each echocardiographic image at the level of mitral valve was displayed in the MATLAB software, all sizes were measured. In each plane and for each frame during diastole to systole, mitral valve annulus and leaflets, bases, middles, and tips of AML and PML were manually identified using MATLAB software. Then, 3D MV annulus geometry, leaflets measurements, and AML and PML positions were automatically computed for each frame and used as input for displacement vectors modeling of the mitral valve leaflets. By solving mathematical equations of inelastic properties of leaflets, each plane was replaced with 60 vectors of displacement and ultimately the mitral valve leaflets were realized by 1125 vectors of displacements, where they also show translations, rotations, and pure strains of bases, middles, and tips of AML and PML simultaneously per cardiac cycle and 
finally, a mitral valve mathematical modeling (Fig 9) $[15,16]$.

\section{CONCLUSIONS}

In spite of the introduced methods applied for echocardiographic datasets at the level of mitral valve for practical applications and clinical verifications, the ideas developed in this article have obvious applications in many areas and the other systems of cardiovascular imaging. The most important applications, however, may be pedagogical. A computer desk (online or offline) can help an echocardiographer to work on the echocardiographic images to evaluate the data.

\section{Conflicts of Interest}

The authors declared no conflict of interest.

\section{REFERENCES}

1. Hossien A, Nithiarasu P, Cheriex E, Maessen J, Sardari Nia P, Ashraf S. A multidimensional dynamic quantification tool for the mitral valve. Interact Cardiovasc Thorac Surg. 2015;21(4):481-7. doi: 10.1093/icvts/ivv187 pmid: 26174119

2. Flachskampf FA, Chandra S, Gaddipatti A, Levine RA, Weyman AE, Ameling W, et al. Analysis of shape and motion of the mitral annulus in subjects with and without cardiomyopathy by echocardiographic 3-dimensional reconstruction. J Am Soc Echocardiogr. 2000;13(4):277-87. pmid: 10756245

3. Salgo IS, Gorman JH, 3rd, Gorman RC, Jackson BM, Bowen FW, Plappert T, et al. Effect of annular shape on leaflet curvature in reducing mitral leaflet stress. Circulation. 2002;106(6):711-7. pmid: 12163432

4. Lee AP, Hsiung MC, Salgo IS, Fang F, Xie JM, Zhang YC, et al. Quantitative analysis of mitral valve morphology in mitral valve prolapse with real-time 3-dimensional echocardiography: importance of annular saddle shape in the pathogenesis of mitral regurgitation. Circulation. 2013;127(7):832-41. doi: 10.1161/CIRCULATIONAHA.112.118083 pmid: 23266859

5. Mahmood F, Subramaniam B, Gorman JH, 3rd, Levine RM, Gorman RC, Maslow A, et al. Three-dimensional echocardiographic assessment of changes in mitral valve geometry after valve repair. Ann Thorac Surg. 2009;88(6):1838-44. doi: 10.1016/j.athoracsur.2009.07.007 pmid: 19932245
6. Maslow A. Mitral valve repair: an echocardiographic review: part 1. J Cardiothorac Vasc Anesth. 2015;29(1):156-77. doi: 10.1053/j.jvca.2014.03.007 pmid: 25043493

7. Roger VL, Go AS, Lloyd-Jones DM, Adams RJ, Berry JD, Brown TM, et al. Heart disease and stroke statistics--2011 update: a report from the American Heart Association. Circulation. 2011;123(4):e18-e209. doi: 10.1161/CIR.0b013e3182009701 pmid: 21160056

8. Nishimura RA, Otto CM, Bonow RO, Carabello BA, Erwin JP, 3rd, Guyton RA, et al. 2014 AHA/ACC guideline for the management of patients with valvular heart disease: a report of the American College of Cardiology/American Heart Association Task Force on Practice Guidelines. J Thorac Cardiovasc Surg. 2014;148(1):e1-e132. doi: 10.1016/j.jtcvs.2014.05.014 pmid: 24939033

9. Gassner E, Ionasec R, Georgescu B, Vogt S, Schoepf U, Comaniciu D. Performance of a Dynamic Aortic Valve Model for Quantification of the Opening Area at Cardiac MDCT Comparison to Manual Planimetry US: Radiological Society of North America; 2008 [cited 2015 Nov 20]. Available from: http://archive.rsna.org/2008/6011906.html.

10. Ionasec RI, Voigt I, Georgescu B, Wang Y, Houle H, VegaHiguera $\mathrm{F}$, et al. Patient-specific modeling and quantification of the aortic and mitral valves from 4-D cardiac CT and TEE. IEEE Trans Med Imaging. 2010;29(9):1636-51. doi: 10.1109/TMI.2010.2048756 pmid: 20442044

11. $\mathrm{Tu} \mathrm{Z}$, editor Probabilistic boosting-tree: Learning discriminative models for classification, recognition, and clustering. Computer Vision, 2005 ICCV 2005 Tenth IEEE International Conference on; 2005: IEEE.

12. Ngernsritraku T, Hur D, McDade K, Kaplan R, Houle H, Lin B, et al. Aortic Annulus Sizing Using A Novel Automated Method: Can Echo Select the Correct Valve Size? US: American Society of Echocardiography; 2015.

13. Alfieri O, Maisano F, De Bonis M, Stefano PL, Torracca L, Oppizzi M, et al. The double-orifice technique in mitral valve repair: a simple solution for complex problems. J Thorac Cardiovasc Surg. 2001;122(4):674-81. doi: $10.1067 / \mathrm{mtc} .2001 .117277$ pmid: 11581597

14. Lancellotti P, Zamorano JL, Vannan MA. Imaging challenges in secondary mitral regurgitation: unsolved issues and perspectives. Circ Cardiovasc Imaging. 2014;7(4):735-46. doi: 10.1161/CIRCIMAGING.114.000992 pmid: 25027455

15. Karvandi M, Ranjbar S, Hassantash SA, Foroughi M. Brief communication: Mathematical concepts of mechanisms of left ventricular myocardium. Int J Med Imaging. 2014;2(2):19-23.

16. Ranjbar S, Karvandi M, Hassantash SA, Foroughi M. How to construct a 3D mathematical/computer model of the left ventricle. Arch Cardiovasc Imaging. 2014;2(2). 